\title{
Miscalculated Lung Shunt Fraction for Planning of Hepatic Radioembolization
}

\author{
Justin S. Caskey ${ }^{1}$, Matthew D. Kay ${ }^{2}$, Natalie A. McMillan², Phillip H. Kuo ${ }^{2,3}$, and Gregory J. Woodhead ${ }^{2}$ \\ ${ }^{1}$ University of Arizona College of Medicine, Tucson, Arizona; ${ }^{2}$ Department of Medical Imaging, University of Arizona College of \\ Medicine, Tucson, Arizona; and ${ }^{3}$ Department of Medicine and Biomedical Engineering, University of Arizona, Tucson, Arizona
}

\begin{abstract}
${ }^{90} \mathrm{Y}$ radioembolization is a safe and efficacious treatment option for many patients with unresectable hepatocellular carcinoma. Potential candidates for radioembolization, based on clinical criteria, undergo ${ }^{99 m}$ Tc-labeled macroaggregated albumin imaging to determine the extent of hepatopulmonary shunting. Dose selection is based on results from shunt imaging and can exclude patients from radioembolization therapy. We present a case of miscalculated lung shunt fraction and the circumstances that led to the critical error.
\end{abstract}

Key Words: ${ }^{90}$ Y radioembolization; lung shunt fraction; $99 \mathrm{mTc}-$ labeled macroaggregated albumin; hepatocellular carcinoma

J Nucl Med Technol 2020; 48:184-186

DOI: $10.2967 /$ jnmt.119.234385

\section{$\mathbf{M}$}

illions of individuals worldwide have hepatocellular carcinoma, the most common primary liver malignancy. In addition to resection, safe and effective treatment options for many patients include transplantation, radiofrequency ablation, chemoembolization, and systemic chemotherapy (1). In ${ }^{90} \mathrm{Y}$ radioembolization, glass or resin beads fixed with the $\beta$-particle-emitting radioisotope ${ }^{90} \mathrm{Y}$ are selectively injected via a microcatheter to deliver high doses of radiation to the tumor. Before treatment, patients undergo important planning studies, including mapping angiography and $99 \mathrm{~m} \mathrm{Tc}-$ labeled macroaggregated albumin ( $\left.{ }^{99 \mathrm{~m}} \mathrm{Tc}-\mathrm{MAA}\right)$ imaging. Information from these studies is used to minimize the risk of nontarget radiation injury to the gastrointestinal tract and lungs (2).

Radiation pneumonitis is a known complication of ${ }^{90} \mathrm{Y}$ radioembolization, and the risk of this complication is related to radiation delivered unintentionally to pulmonary tissue via hepatopulmonary shunting (3). ${ }^{99 \mathrm{~m} T c-M A A}$ imaging is therefore critical for ${ }^{90} \mathrm{Y}$ patient selection, a requirement for shunt reduction intervention and radioembolization

Received Jul. 29, 2019; revision accepted Oct. 7, 2019.

For correspondence or reprints contact: Matthew D. Kay, Department of Medical Imaging, University of Arizona College of Medicine, $1501 \mathrm{~N}$. Campbell Ave., Tucson, AZ 85719.

E-mail: mkay@radiology.arizona.edu

Published online Dec. 6, 2019.

COPYRIGHT (c) 2020 by the Society of Nuclear Medicine and Molecular Imaging. dosing. After ${ }^{99 m}$ Tc-MAA injection, anterior and posterior planar imaging is performed. The geometric mean is calculated as the square root of the product of counts for regions of interest (ROIs) from anterior and posterior planar images (Fig. 1). Many vendors' software generates a single geometric mean image, which is a composite of the anterior and (flipped) posterior images, and from this the geometric mean counts for each ROI are obtained. The lung shunt fraction (LSF) is calculated as the counts from the lung ROI divided by the total counts for the lung and liver ROI (from the geometric mean image) (Fig. 1). For radioembolization therapy, an LSF of more than $20 \%$ or an LSF that results in an estimated lung radiation exposure of more than $30 \mathrm{~Gy}$ (based on the planned ${ }^{90} \mathrm{Y}$ dose) is considered a contraindication (4) or requires a significant dose reduction at the risk of reduced treatment efficacy.

\section{CASE REPORT}

A 65-y-old man with multifocal hepatocellular carcinoma (Fig. 2) underwent evaluation for potential treatment with ${ }^{90} \mathrm{Y}$ radioembolization. Pretreatment ${ }^{99 \mathrm{~m} T c-M A A}$ imaging was performed to assess hepatopulmonary shunting. ROI determination and postprocessing were completed per standard procedures, and the LSF was initially calculated as $29.5 \%$ (Fig. 3A). The nuclear medicine physician noted a potential error in shunt fraction based on visual assessment and requested that the image be reprocessed. After reprocessing, the LSF was recalculated as $7.9 \%$ (Fig. 3B). The patient ultimately received a successful ${ }^{90} \mathrm{Y}$ radioembolization.

\section{DISCUSSION}

This case highlights a critical source of error that can occur during LSF calculation by ${ }^{99 \mathrm{~m}} \mathrm{Tc}-\mathrm{MAA}$ imaging.

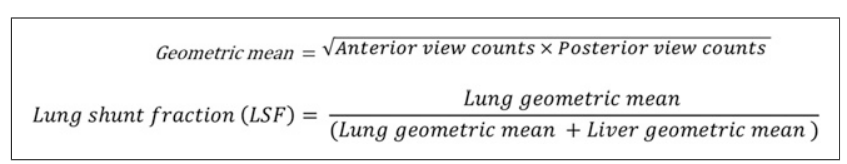

FIGURE 1. Formulas used for calculating geometric mean (for designated ROI) and LSF. 


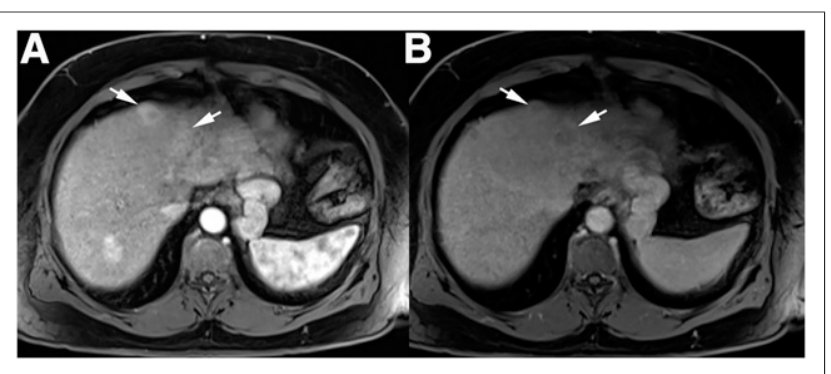

FIGURE 2. Contrast-enhanced axial T1-weighted images during arterial $(A)$ and delayed $(B)$ phases demonstrate washout within 2 adjacent hepatocellular carcinomas (arrows) located anteriorly within segment $4 \mathrm{~A}$ of liver.

Initially, the nuclear medicine technologist mistakenly labeled the raw data from a posterior flipped view as the geometric mean image; thus, the ROI contained counts from only the posterior view. The initial incorrect LSF (using only the flipped posterior planar image) was $159,7289 \div(159,7289+$ $380,618)=0.295$ (Fig. 3A). The correct LSF (based on counts from the geometric mean image) was $282,930 \div$ $(282,930+3,284,000)=0.079$ (Fig. 3B).

The initial shunt fraction of $29.5 \%$ would be a contraindication to ${ }^{90} \mathrm{Y}$ radioembolization, and the error thus would have mistakenly precluded the patient from therapy. Although differences in ROI size can alter count totals, the variance in this case was too large to be attributed to this factor alone because the error led to a 3.5 greater discrepancy between the calculated LSFs. The initial artifactually low liver count was especially exacerbated by the anterior position of the tumor (Fig. 4), as only the (flipped) posterior planar image was used for the initial LSF calculation. Our nuclear medicine laboratory has scanners from multiple vendors, and this error likely occurred because of confusion over how the geometric mean is displayed or calculated among different vendors.

To overcome this potential error, the nuclear medicine technologist may benefit from having easy access to a vendorspecific guide for the accurate calculation of LSF. Labeling

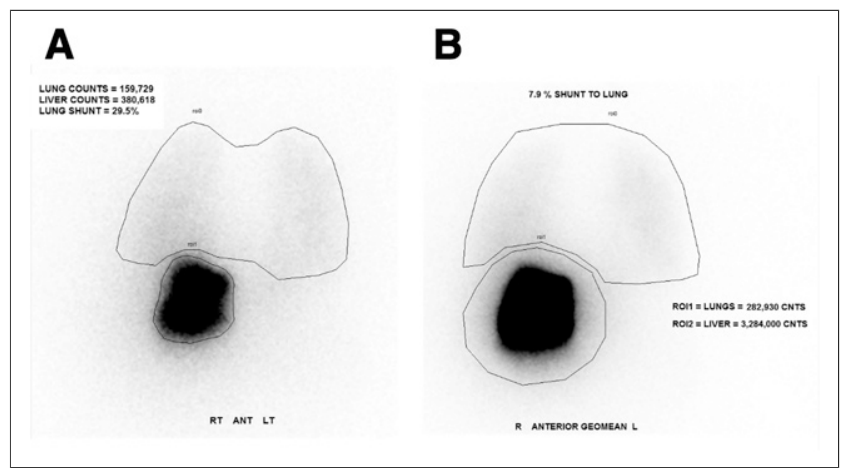

FIGURE 3. (A) Initial 99mTc-MAA (flipped) posterior planar image with lung and liver ROls. Incorrect LSF calculated was $29.5 \%$. (B) 99mTc-MAA geometric mean image with lung and liver ROls. Correct LSF calculated was $7.9 \%$.

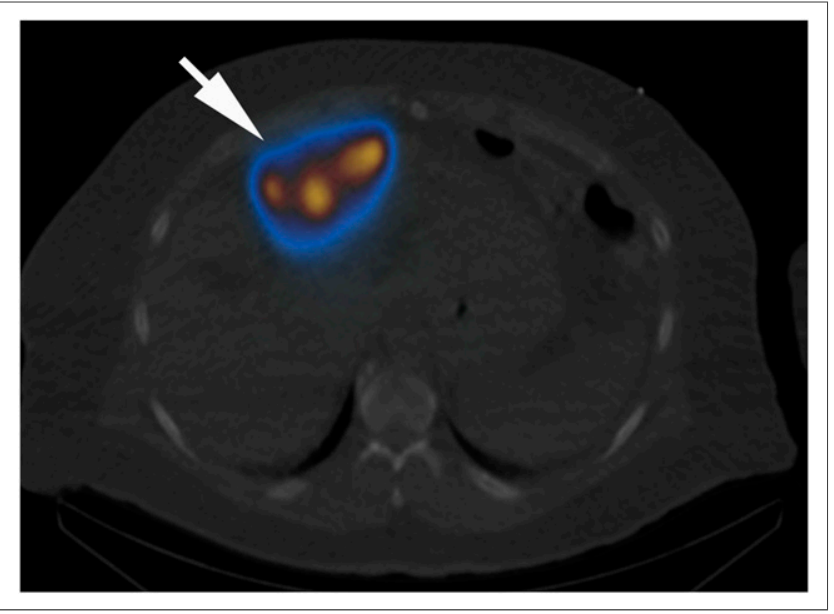

FIGURE 4. Selected axial 99mTc-MAA SPECT/CT image confirming radiotracer delivery to anteriorly positioned hepatocellular carcinomas (arrow).

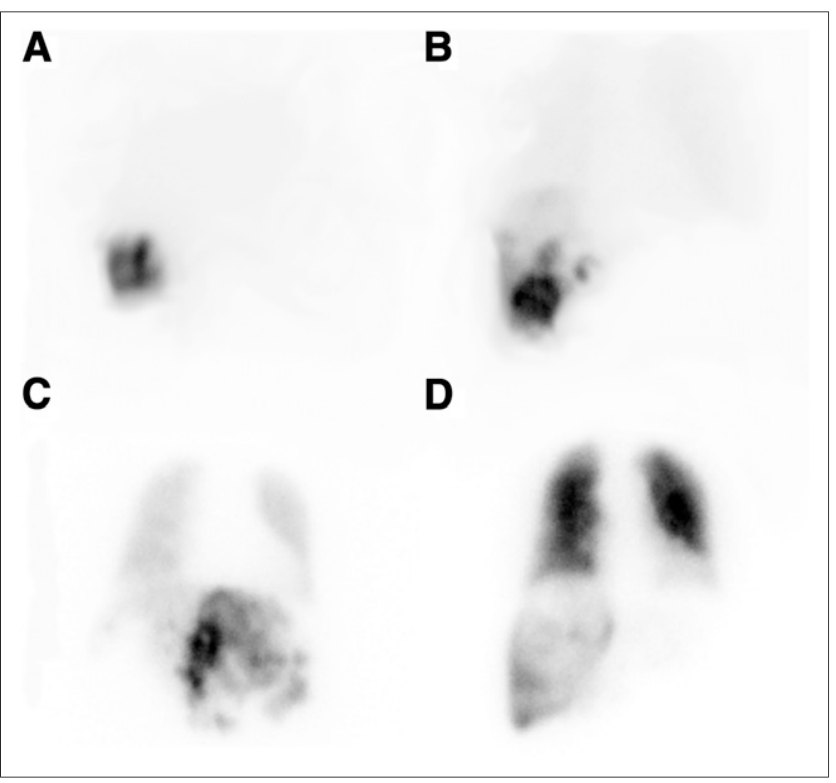

FIGURE 5. Lung shunt fraction visual reference for LSFs of $3 \%(A), 15 \%(B), 37 \%(C)$, and $79 \%(D)$.

the images with "geometric mean" instead of "ROI" or "counts," as in our case (Fig. 3), may act as a reminder to use the correct data source. Further, if the nuclear medicine technologist (or nuclear medicine physician) needs to actively window the raw data to discriminate the lung ROI (or liver ROI) from the background, then the LSF should be low. Nuclear medicine technologists and nuclear medicine physicians may also use the provided LSF visual reference (Fig. 5) as a guide when visually assessing the adequacy of the LSF from the raw data images.

\section{CONCLUSION}

99mTc-MAA imaging and LSF calculations are important steps in pretreatment assessment for patients before 
${ }^{90} \mathrm{Y}$ radioembolization for hepatocellular carcinoma. Confirming that the visual assessment and calculated LSF are concordant ensures that the LSF has been calculated appropriately from the geometric mean image during processing.

\section{DISCLOSURE}

No potential conflict of interest relevant to this article was reported.

\section{REFERENCES}

1. Saini A, Wallace A, Alzubaidi S, et al. History and evolution of yttrium-90 radioembolization for hepatocellular carcinoma. J Clin Med. 2019;8:55.

2. Leung WT, Lau WY, Ho SK, et al. Measuring lung shunting in hepatocellular carcinoma with intrahepatic-arterial technetium-99m macroaggregated albumin. J Nucl Med. 1994;35:70-73.

3. Leung TW, Lau WY, Ho SK, et al. Radiation pneumonitis after selective internal radiation treatment with intraarterial ${ }^{90}$ yttrium-microspheres for inoperable hepatic tumors. Int J Radiat Oncol Biol Phys. 1995;33:919-924.

4. Coldwell D, Sangro B, Wasan H, Salem R, Kennedy A. General selection criteria of patients for radioembolization of liver tumors: an international working group report. Am J Clin Oncol. 2011;34:337-341. 